\title{
Dabigatran Versus Acetylsalicylic Acid for Secondary Stroke Prevention in Patients with Embolic Stroke of Undetermined Source: Rationale, Design and Baseline Data from RE-SPECT ESUS
}

H-C Diener ${ }^{1}$, JD Easton ${ }^{2}$, CB Granger ${ }^{3}$, M Brueckmann ${ }^{4}$, L Cronin ${ }^{5}$, C Grauer 6 , D Cotton ${ }^{7}$, RL Sacco ${ }^{8}$ on behalf of the RE-SPECT ESUS ${ }^{\text {Tm }}$ Investigators 'University Hospital, Essen, Germany; ${ }^{2}$ University of California-San Francisco, San Francisco, CA, USA; ${ }^{3}$ Duke Clinical Research Institute, Durham, NC, USA;

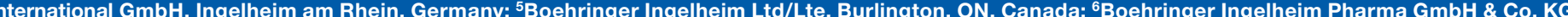
Biberach, Germany; ${ }^{7}$ Boehringer Ingelheim Pharmaceuticals, Inc., Ridgefield, CT, USA; ${ }^{8}$ University of Miami, Miami, FL, USA

\section{BACKGROUND}

Ischaemic strokes account for approximately $92 \%$ of all strokes, and around $25 \%$ of ischaemic strokes are cryptogenic., ${ }^{1,2}$

- 'Embolic Strokes of Undetermined Source (ESUS)' are a subset of cryptogenic strokes defined as non-lacunar infarcts, without relevant arterial stenoses or cardiac sources, with no other specific known cause ${ }^{2}$

- Antiplatelet agents, such as acetylsalicylic acid, are the standard of care for the prevention of secondary stroke in patients with cryptogenic stroke. ${ }^{3}$

- The optimal antithrombotic treatment for secondary stroke prevention in patients with ESUS has not been established.

The direct oral anticoagulant dabigatran etexilate is commonly used for stroke prevention in patients with atrial fibrillation (AF) ${ }^{4}$ and may also be effective for the prevention of recurrent stroke in patients with ESUS.

\section{OBJECTIVE}

- The RE-SPECT ESUS'M trial will compare the efficacy and safety of dabigatran etexilate with acetylsalicylic acid for secondary stroke prevention in patients with ESUS.

\section{METHODS}

Study Design

- RE-SPECT ESUS is a large-scale, phase III, double-blind, randomized trial.

C Patients were randomized 1:1 to receive dabigatran etexilate $150 \mathrm{mg}$ twice daily (or $110 \mathrm{mg}$ twice daily in those aged $\geq 75$ years or with creatinine clearance $\geq 30$ to $<50 \mathrm{~mL} / \mathrm{min}$ ) or acetylsalicylic acid $100 \mathrm{mg}$ once daily (Figure 1 ).

- Patients were treated for a minimum of 6 months and for up to approximately 3 years.

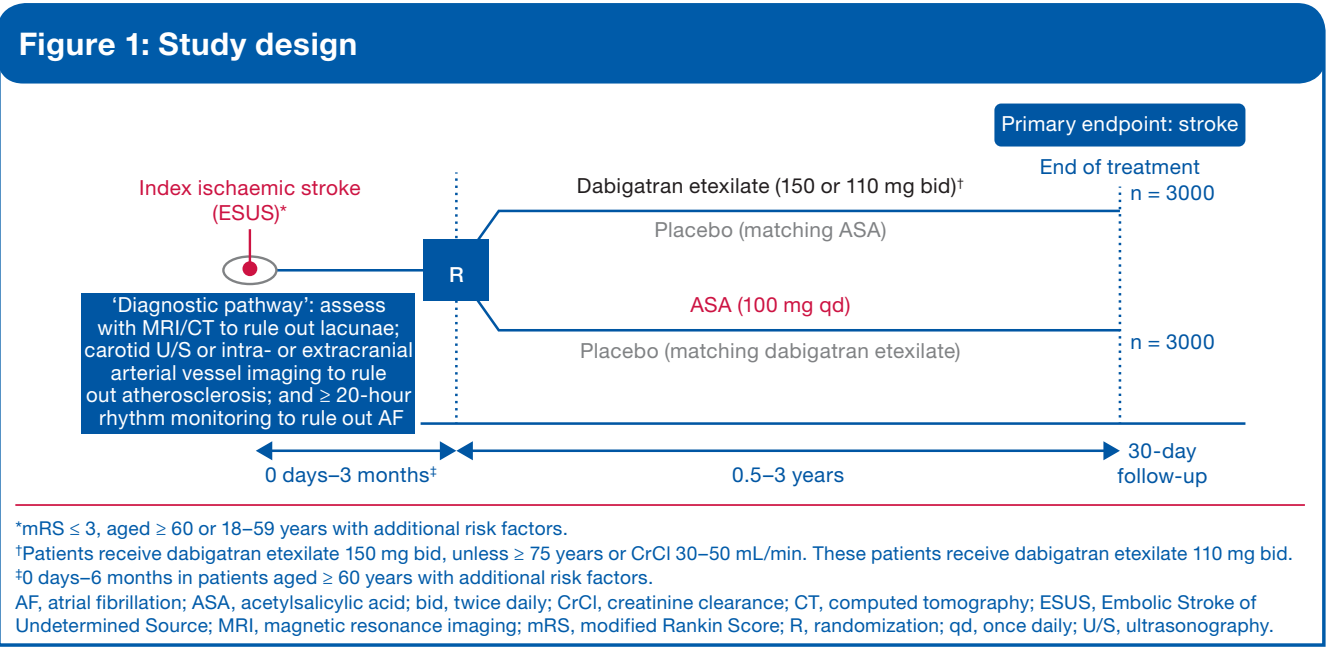

Study Participants

C The main inclusion and exclusion criteria are shown in Table 1.

\section{Table 1: Main inclusion and exclusion criteria}

\begin{tabular}{ll} 
Inclusion criteria & Exclusion criteria \\
\hline ESUS diagnosed within 3 months prior to randomization & Modified Rankin Score $\geq 4$ \\
(6 months in patients aged $\geq 60$ years with at least & Major-risk cardioembolic source of embolism \\
one additional risk factor for recurrent stroke) & Any indication for anticoagulation \\
Modified Rankin Score $\leq 3$ & Other specific stroke aetiology \\
Patients aged $\geq 60$ years, or $18-59$ years with & Primary intracerebral haemorrhage on qualifying \\
a stroke risk factor such as: & neuroimaging \\
- Mild to moderate symptomatic heart failure & History of AF \\
- Diabetes mellitus & Conditions associated with increased bleeding risk \\
- Hypertension & Symptomatic non-traumatic intracranial haemorrhage \\
- Patent foramen ovale & and renal impairment (creatinine clearance $<30 \mathrm{~mL} / \mathrm{min})$ \\
- Prior stroke or transient ischaemic attack & \\
- $\mathrm{CHA}_{2} \mathrm{DS} \mathrm{DS}_{2}-\mathrm{VASC} \geq 3$ & \\
\hline $\mathrm{AF}$, atrial fibrillation; ESUS, Embolic Stroke of Undetermined Source. & \\
\hline
\end{tabular}

Study Endpoints

- The main efficacy and safety endpoints are presented in Table 2.

\section{Table 2: Efficacy and safety endpoints}

\begin{tabular}{lll} 
Primary efficacy endpoint & Key secondary endpoints & Main safety endpoint \\
\hline Time to first recurrent stroke & Ischaemic stroke & Time to first major bleed \\
(ischaemic, haemorrhagic or & Composite endpoint of non-fatal & \\
unspecified) & stroke, non-fatal Ml and CV death & \\
\hline $\mathrm{CV}$, cardiovascular; Ml, myocardial infarction. &
\end{tabular}

\section{Statistical Analysis}

- The trial was event-driven and would complete once at least 353 stroke events had been reported.

The trial was powered to detect superiority. With 353 events the trial has approximately $92 \%$

power to show a $30 \%$ relative risk reduction, based on a yearly stroke rate of approximately $4.1 \%$ with acetylsalicylic acid.

- Originally 6000 patients were expected to be enrolled and the observational period was anticipated to be approximately 3 years. However, these were dependent on the actual event rate.

- Time-to-event endpoints will be analysed using the Cox proportional hazards regression model.

\section{STUDY PROGRESS}

C The study was initiated in December 2014. Randomization was completed in January 2018 Thich time 5390 patients had been randomized.

- Patients were recruited from over 40 countries shown in Figure 2.

\section{Figure 2: Geographical distribution of participating countries}

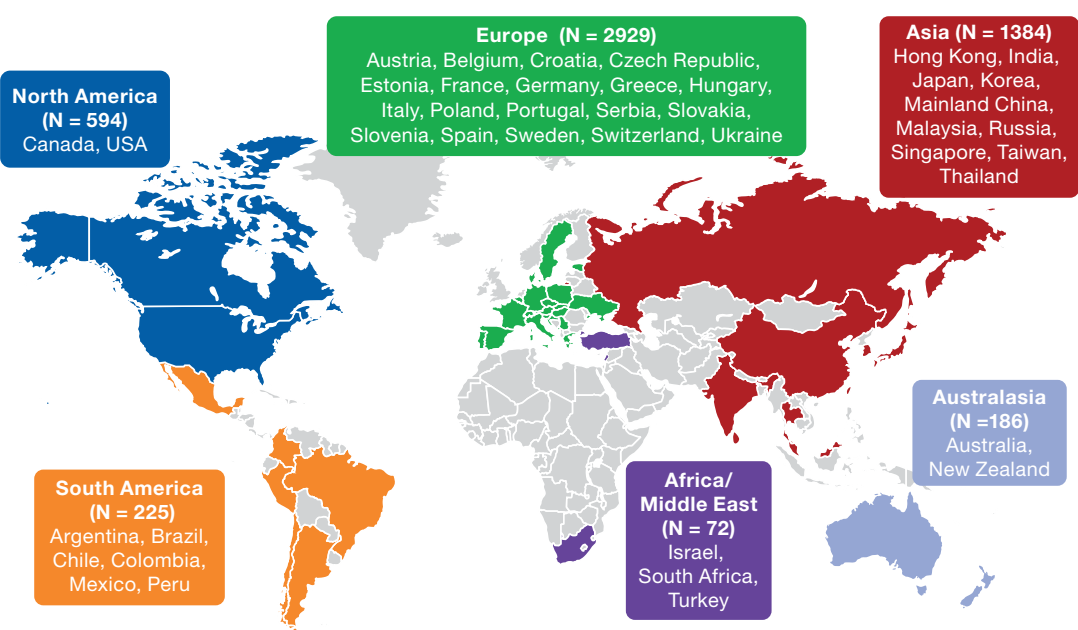

Patient Characteristics

C Data regarding baseline characteristics are available for 5390 patients (Table 3).

C Approximately $63 \%$ of patients were male.

- The median time from stroke to study entry was 44 days; the duration of cardiac monitoring before inclusion into the trial was $20-48$ hours in $79 \%$ of patients; $>48-72$ hours in $6 \%$ of patients and $>72$ hours -7 days in $5 \%$ of patients.

\begin{tabular}{lc|}
\hline \multicolumn{2}{|l|}{ Table 3: Baseline characteristics (data updated 19 April, 2018) } \\
\hline Number of patients, $\mathrm{n}$ & 5390 \\
Mean age, years & $64.2(11.4)$ \\
Male, \% & 63 \\
Median time to study entry, days (IQR) & $44(21-80)$ \\
$\mathrm{CHA}_{2} \mathrm{DS}_{2}-\mathrm{VASc}$ score, mean (SD) & $4.3(1.3)$ \\
$\mathrm{CHA}_{2} \mathrm{DS}_{2}$-VASc scores, \% & \\
2 & 6 \\
3 & 26 \\
4 & 28 \\
5 & 23 \\
$\geq 6$ & 18 \\
TIA or stroke prior to index event, \% & 18 \\
History of hypertension, \% & 74 \\
Diabetes mellitus, \% & 23 \\
Patent foramen ovale, \% & 13 \\
Coronary artery disease, \% & 11 \\
LV dysfunction/CHF, \% & 5 \\
\hline CHF, congestive heart failure; lQR, Interquartile range; LV, left ventricular; SD, standard deviation; TIA, transient ichaemic attack. \\
\hline
\end{tabular}

\section{CONCLUSIONS}

- Efficacy and safety data from the RE-SPECT ESUS trial will help to determine whether dabigatran is superior to acetylsalicylic acid for secondary stroke prevention in patients with ESUS.

- These findings will aid physicians to optimally treat patients with ESUS.

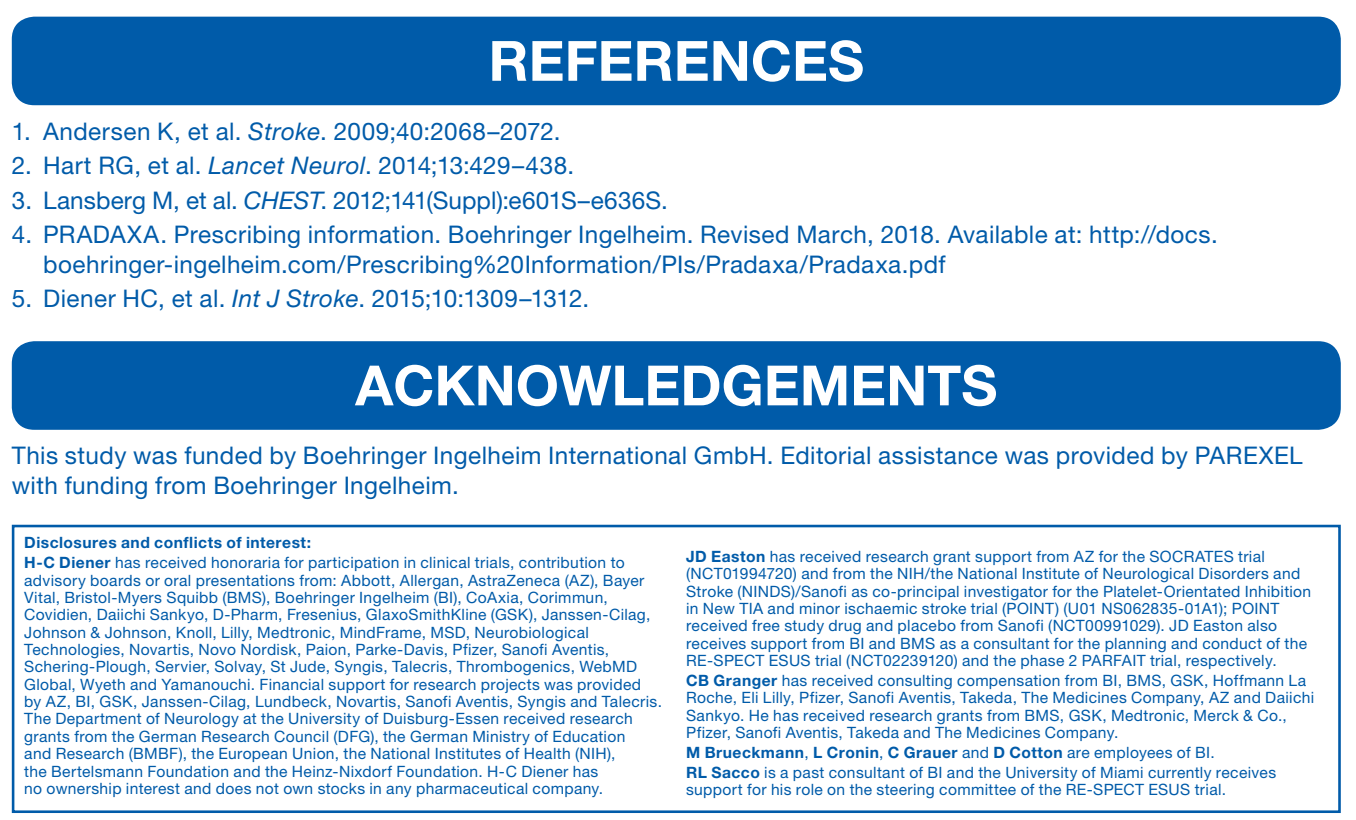

口秝解 Copies of this poster can be obtained through this QR (Quick Response) code. 